Discussion Paper No. 646

\title{
VICKEREY ALLOCATION RULE WITH INCOME EFFECT
}

\author{
Hiroki Saitoh \\ and \\ Shigehiro Serizawa
}

December 2005

The Institute of Social and Economic Research Osaka University

6-1 Mihogaoka, Ibaraki, Osaka 567-0047, Japan 


\title{
Vickrey Allocation Rule with Income Effect ${ }^{1}$
}

\author{
HIROKI SAITOH \\ Institute of Social and Economic Research, Osaka University \\ 6-1, Mihogaoka, Ibaraki, Osaka 567-0047, JAPAN \\ AND \\ SHIGEHIRO SERIZAWA \\ Institute of Social and Economic Research, Osaka University \\ 6-1, Mihogaoka, Ibaraki, Osaka 567-0047, JAPAN \\ E-mail: serizawa@iser.osaka-u.ac.jp \\ Phone: (+81) 6-6879-8558 \Fax: (+81) 6-6878-2766
}

December 21, 2005

Keywords: Vickrey auction, allocation rule, income effect, strategy-proofness JEL Classification Numbers: D44, C78, D71, D61, D82

We consider situations where a society tries to efficiently allocate several homogeneous and indivisible goods among agents. Each agent receives at most one unit of the good. For example, suppose that a government wishes to allocate a fixed number of licenses to operate in its country to private companies with highest abilities to utilize the licenses. Usually companies with higher abilities can make more profits by licenses and are willing to pay higher prices for them. Thus, auction mechanisms are often employed to extract the information on companies' abilities and to allocate licenses efficiently. However, if prices are too high, they may damage companies' abilities to operate. Generally high prices may change the benefits agents obtain from the goods unless agents' preferences are quasi-linear, and we call it "income effect". In this paper, we establish that on domains including nonquasi-linear preferences, that is, preferences exhibiting income effect, an allocation rule which satisfies Pareto-efficiency, strategy-proofness, individual rationality, and nonnegative payment uniquely exists and it is the Vickrey allocation rule.

\footnotetext{
${ }^{1}$ This article is dedicated to the memory of the late Jennifer Mao, who motivated Serizawa to analyze the problem addressed in this article. We thank the participants of the Seventh International Meeting of Social Choice and Welfare (2004, July) and Rokko Forum of Kobe University (2005 December) for their useful comments on the first version, and we also thank Professor Masaki Aoyagi for his helpful comments.
} 


\section{INTRODUCTION}

We consider situations where a society tries to efficiently allocate $m$ homogeneous and indivisible goods among $n$ agents. Each agent receives at most one unit of the good. For example, suppose that a government wishes to allocate a fixed number of licenses to operate in its country to private companies with highest abilities to utilize the licenses ${ }^{2}$. Usually companies with higher abilities can make more profits by licenses and are willing to pay higher prices for them. Thus, auction mechanisms are often employed to extract the information on companies' abilities and to allocate licenses efficiently. However, if prices are too high, they may damage companies' abilities to operate. Generally high prices may change the benefits agents obtain from the goods unless agents' preferences are quasi-linear ${ }^{3}$, and we call it "income effect". The society must take this effect into account when it tries to efficiently allocate the goods. In this paper, we study rules allocating goods efficiently even if income effect exists.

An allocation rule is generally formulated as a function from the set of possible agents' preference profiles to the feasible set, and the set of possible agents' preference profiles is called "domain". Given an allocation rule, since agents' private preferences are not known to the others, there may be incentives for agents to misrepresent their preferences in order to manipulate the final outcomes to their favor. As a result, the actual outcomes may not constitute an efficient allocation relative to agents' true preferences. Thus allocation rules need to be immune to such strategic misrepresentation in order to surely attain an efficient allocation for agents' true preferences. If an allocation rule is immune to strategic behavior by any agent, that is, if it is a dominant strategy for each agent to announce his true preferences, then the allocation rule is said to be strategy-proof. To induce agents' voluntary participation, it is desirable for allocation rules to satisfy the property of individual rationality; it never assigns an allocation which makes some agent worse off than he would be if he receives no good and pays nothing. Nonnegative payment is also a natural requirement; it says that agents' payment should not be negative. Thus it is important to know what allocation rules satisfy Pareto-efficiency, strategy-proofness, individual rationality, and nonnegative payment.

The Vickrey allocation rule is the rule originally defined on the quasi-linear domain ${ }^{4}$ such that agents with $m$ highest valuations ${ }^{5}$ of the goods receive the goods and pay the $(m+1)$-th valuation, and other agents pay nothing. The Vickrey allocation rule can be generalized to domains including nonquasi-linear preferences, that is, preferences exhibiting income effect. In this paper, we establish that on these domains, an allocation rule which satisfies Pareto-efficiency, strategy-proofness, individual rationality, and nonnegative payment uniquely exists and it is the Vickrey allocation rule.

Holmstrom (1979) studies public good models, and establish that an allocation rule is strategy-proof and provides the efficient level of the public good on the quasi-linear domain if and only if it is a Groves rule. The Vickrey allocation rule is the counterpart of the Groves rule in the auction model. Holmstrom's (1979) results can be also applied

\footnotetext{
${ }^{2}$ The frequency auctions recently implemented in many countries are such examples. See Klemperer (2001) for the details.

${ }^{3} \mathrm{~A}$ preference is quasi-linear if the benefits from consuming the good are independent of payments.

${ }^{4}$ The quasi-linear domain is the class of quasi-linear preference profiles

5If agents' preference are quasi-linear, since their benefits from consuming the good are independent of payments, their valuations of the goods are well-defined.
} 
to auction models when agents have only quasi-linear preferences, and imply the same conclusion as ours. ${ }^{6}$

However, there are various cases in which preferences are not quasi-linear. In the case where agents are households, as the prices of goods are higher, they can afford to purchase less complements, and benefit less from the goods. Unless the prices of goods are small enough compared to their incomes, this effect will give rise to innegligible degree of nonquasi-linearity. The vehicle ownership license auction in Singapore is also one of such examples, where license prices are often as high as the annual income of average households. In the case where agents are private firms in auctions, high prices bring them difficulties in raising the funds to invest for the effective uses of the auctioned items. Che and Gale (1998) demonstrate that the imperfection of financial markets causes agents to have nonquasi-liner preferences by citing a variety of examples. Holmstrom's (1979) results do not cover these cases while our results do.

Whether an allocation rule satisfies certain requirements or not depends on its domain. A conclusion on one domain does not simply imply the same conclusion on other domains. Even though the same conclusion happens to hold on different domains, the same proof technique may not be applied to different domains. We emphasize that Holmstrom's (1979) proof technique does not work on domains including nonquasi-linear preferences. ${ }^{7}$ When preferences are quasi-linear, a Pareto-efficient allocation of goods is uniquely determined by net benefit maximization. Owing to this fact, Holmstrom (1979) actually characterizes payment rules satisfying his requirements. On the other hand, when preferences are not quasi-linear, whether an allocation of goods is Pareto-efficient or not depends on how much agents pay, and therefore, both of the allocation of goods and payments need to be analyzed simultaneously.

Demange, Gale and Sotomyer (1986) and Roth and Sotomyer (1990) also study the strategy-proofness of Vickrey auction with mulitple units. Strengthing their results, Miyake (1998) shows that the Vickrey allocation rule is the unique allocation rule among "competitive allocation rules" 8 . Our characterization of the Vickrey allocation rule is more general in the sense that we show the uniques among the general class of allocation rules. Recently and independently Sakai (2005) establishes the same result as ours in the case in which there is one unit of the auctioned good.

Section 2 sets up the model, defines basic notions, and states main results. Section 3 provides the proofs of our main results.

\section{The Model and Main Results}

We denote the set of agents by $N=\{1,2, \ldots, n\}(n \geq 2)$. There are $m<n$ indivisible and homogeneous goods to be allocated among agents. Each agent is admitted to receive

\footnotetext{
${ }^{6}$ Similar characterizations of Groves rules are previously established by Green and Laffont (1977), and Walker (1978). However, the characterizations of the these two articles cannot be applied to auction models since they assume that the class of admissible preferences include preferences which are not admissible in auction models. Makowski and Ostroy (1987) apply Holmstrom's (1979) result to more general model, and obtain similar conclusion when preferences are quasi-linear. Chew and Serizawa (2004) also characterize the Vickrey allocation rule in the auction model with quasi-linear domains by induction logic.

${ }^{7}$ See Milgrom (2004) for the detailed explanation of Holmtrom's technique.

${ }^{8} \mathrm{An}$ allocation rule is a competitive allocation rule if the rule assigns to each preference profile a competitive equilibrium allocation for the profile.
} 
at most one unit of the good. A good allocation is a $n$-tuple $a=\left(a^{1}, \ldots, a^{n}\right) \in\{0,1\}^{n}$ such that $\sum a^{i}=m$. We denote the set of good allocations by $A$. We denote the money agent $i$ pays by $t^{i} \in \mathbb{R}$. A feasible allocation is a pair $z=(a ; t) \in A \times \mathbb{R}^{n}$, where $t=\left(t^{1}, \ldots, t^{n}\right) .{ }^{9}$ We denote the set of feasible allocations by $Z$.

Agents have preferences on $\{0,1\} \times \mathbb{R}$. We abuse language and identify a preference with its utility representation $u^{i}$.

Definition: A preference $u^{i}$ on $\{0,1\} \times \mathbb{R}$ is classical if it satisfies the following properties:

(Continuity) For all $a^{i} \in\{0,1\}, u^{i}\left(a^{i}, t^{i}\right)$ is continuous with respect to $t^{i}$.

(Strict Monotonicity) For all $a^{i} \in\{0,1\}$, all $t^{i} \in \mathbb{R}$, and all $\widehat{t}^{i} \in \mathbb{R}$,

$$
u^{i}\left(1, t^{i}\right)>u^{i}\left(0, t^{i}\right) \quad \& \quad\left[\widehat{t}^{i}>t^{i} \Rightarrow u^{i}\left(a^{i}, t^{i}\right)>u^{i}\left(a^{i}, \widehat{t}^{i}\right)\right] .
$$

(Finiteness) For all $t^{i} \in \mathbb{R}$, there exist $d_{1} \in \mathbb{R}_{++}$and $d_{2} \in \mathbb{R}_{++}$such that $u^{i}\left(0, t^{i}\right) \geq$ $u^{i}\left(1, t^{i}+d_{1}\right)$ and $u^{i}\left(0, t^{i}-d_{2}\right) \geq u^{i}\left(1, t^{i}\right)$.

We denote the class of agent $i$ 's classical preferences by $U_{C}^{i}$, and $U_{C}=U_{C}^{1} \times \cdots \times U_{C}^{n}$.

Definition: A classical preference $u^{i}$ on $\{0,1\} \times \mathbb{R}$ is quasi-linear if there is a value $v^{i} \in \mathbb{R}_{++}$such that for all $a^{i} \in\{0,1\}$ and all $t^{i} \in \mathbb{R}, u^{i}\left(a^{i}, t^{i}\right)=v^{i} \cdot a^{i}-t^{i}$.

We denote the class of agent $i$ 's quasi-linear preferences by $U_{L}^{i}$, and $U_{L}=U_{L}^{1} \times \cdots \times U_{L}^{n}$.

Examples below illustrate the situations in which agents have nonquasi-linear preferences.

EXAmPle 1: Nonlinear cost of payments. Suppose that agent $i$ has budget $I^{i}$, and he has to incur interst cost if his payment exceeds his budget. Then, his cost $c^{i}\left(t^{i}\right)$ of payment $t^{i}$ is $t^{i}$ up to $I^{i}$ and is $I^{i}+(1+r) \cdot\left(t^{i}-I^{i}\right)$ above $I^{i}$, where $r>0$ is a interest rate. Given a value $v^{i}$ and a budget $I^{i}$, agent $i$ 's utility function $u^{i}\left(\cdot, \cdot ; v^{i}, I^{i}\right)$ is the function on $\{0,1\} \times \mathbb{R}$ such that for all $a^{i} \in\{0,1\}$ and all $t^{i} \in \mathbb{R}, u^{i}\left(a^{i}, t^{i} ; v^{i}, I^{i}, c^{i}\right)=v^{i} \cdot a^{i}-c^{i}\left(t^{i}\right)$. Unless $I^{i}=\infty, u^{i}\left(\cdot, \cdot ; v^{i}, I^{i}\right)$ is not quasi-linear.

Example 2: Surplus depending values. Suppose that agent $i$ has budget $I^{i}$ and his value of the good depends on the surplus $m^{i}=I^{i}-t^{i}$. For instance, imagine that the good is a license and the agent can use the license more effectively with more surplus, or that the agent benefits more from the good with more surplus since he can puchase more complements by the surplus. The agent has access to credit, and the surplus $m^{i}=I^{i}-t^{i}$ can be negative. Then, his value $v^{i}$ of the good is a function from $\mathbb{R}$ to $\mathbb{R}$. Assume that the value function $v^{i}: \mathbb{R} \rightarrow \mathbb{R}$ is continuous. Given a value function $v^{i}$ and a budget $I^{i} \in \mathbb{R}$, agent $i$ 's utility function $u^{i}\left(\cdot, \cdot ; v^{i}, I^{i}\right)$ is the function on $\{0,1\} \times \mathbb{R}$ such that for all $a^{i} \in\{0,1\}$ and all $t^{i} \in \mathbb{R}, u^{i}\left(a^{i}, t^{i} ; v^{i}, I^{i}\right)=v^{i}\left(I^{i}-t^{i}\right) \cdot a^{i}+I^{i}-t^{i}$. Unless $v^{i}: \mathbb{R} \rightarrow \mathbb{R}$ is a constant function, $u^{i}\left(\cdot, \cdot ; v^{i}, I^{i}\right)$ is not quasi-linear.

Given $u^{i} \in U_{C}^{i}$ and $t^{i} \in \mathbb{R}$, we define willing to pay $W P\left(u^{i}, t^{i}\right) \in \mathbb{R}$ by $u^{i}\left(1, t^{i}+\right.$ $\left.W P\left(u^{i}, t^{i}\right)\right)=u^{i}\left(0, t^{i}\right)$, and compensating transfer $C T\left(u^{i}, t^{i}\right) \in \mathbb{R}$ by $u^{i}\left(0, t^{i}-C T\left(u^{i}, t^{i}\right)\right)=$ $u^{i}\left(1, t^{i}\right)$. Note that $u^{i} \in U_{C}^{i}$ implies (i) the existences of $W P\left(u^{i}, t^{i}\right)$ and $C T\left(u^{i}, t^{i}\right)$ for all $t^{i} \in \mathbb{R}$, that continuity of $u^{i}$ implies (ii) $W P\left(u^{i}, t^{i}\right)$ and $C T\left(u^{i}, t^{i}\right)$ are continuous with respect to $t^{i}$, and that strict monotonicity implies (iii) $W P\left(u^{i}, t^{i}\right)>0$ and $C T\left(u^{i}, t^{i}\right)>0$ for all $t^{i} \in \mathbb{R}$. Also note that $C T\left(u^{i}, t^{i}+W P\left(u^{i}, t^{i}\right)\right)=W P\left(u^{i}, t^{i}\right)$ and $W P\left(u^{i}, t^{i}-C T\left(u^{i}, t^{i}\right)\right)=C T\left(u^{i}, t^{i}\right)$ for all $t^{i} \in \mathbb{R}$. Thus, (iv) $W P\left(u^{i}, t^{i}\right)$ is increasing in $t^{i}$ if and only if $C T\left(u^{i}, t^{i}\right)$ is increasing in $t^{i}$, (v) $W P\left(u^{i}, t^{i}\right)$ is decreasing in $t^{i}$ if and only if

\footnotetext{
${ }^{9}$ Since $t \in \mathbb{R}^{n}$, negative payments are admissible for feasible allocations.
} 
$C T\left(u^{i}, t^{i}\right)$ is decreasing in $t^{i}$, and (vi) $W P\left(u^{i}, t^{i}\right)$ is constant in $t^{i}$ if and only if $C T\left(u^{i}, t^{i}\right)$ is constant in $t^{i}$.

The values of willing to pay are the maximum prices agents demand for the good. Income effect is the change of such values as the result of income change.

Definition: A classical preference $u^{i}$ on $\{0,1\} \times \mathbb{R}$ exhibits positive income effect if for all $t^{i} \in \mathbb{R}$ and all $\widehat{t^{i}} \in \mathbb{R}, t^{i}<\widehat{t^{i}}$ implies $W P\left(u^{i}, t^{i}\right)>W P\left(u^{i}, \widehat{t}^{i}\right)$.

DeFinition: A classical preference $u^{i}$ on $\{0,1\} \times \mathbb{R}$ exhibits nonnegative income effect if for all $t^{i} \in \mathbb{R}$ and all $\widehat{t^{i}} \in \mathbb{R}, t^{i} \leq \widehat{t^{i}}$ implies $W P\left(u^{i}, t^{i}\right) \geq W P\left(u^{i}, \widehat{t}^{i}\right)$.

Definition: A classical preference $u^{i}$ on $\{0,1\} \times \mathbb{R}$ exhibits negative income effect if for all $t^{i} \in \mathbb{R}$ and all $\widehat{t^{i}} \in \mathbb{R}, t^{i}<\widehat{t}^{i}$ implies $W P\left(u^{i}, t^{i}\right)<W P\left(u^{i}, \widehat{t}^{i}\right)$.

Definition: A classical preference $u^{i}$ on $\{0,1\} \times \mathbb{R}$ exhibits nonpositive income effect if for all $t^{i} \in \mathbb{R}$ and all $\widehat{t^{i}} \in \mathbb{R}, t^{i} \leq \widehat{t^{i}}$ implies $W P\left(u^{i}, t^{i}\right) \leq W P\left(u^{i}, \widehat{t}^{i}\right)$.

We denote the class of agent $i$ 's preferences exhibiting nonnegative income effect by $U_{+}^{i}$, and $U_{+}=U_{+}^{1} \times \cdots \times U_{+}^{n}$. We denote the class of agent $i$ 's preferences exhibiting positive income effect by $U_{++}^{i}$, and $U_{++}=U_{++}^{1} \times \cdots \times U_{++}^{n}$. We denote the class of agent $i$ 's preferences exhibiting nonpositive income effect by $U_{-}^{i}$, and $U_{-}=U_{-}^{1} \times \cdots \times U_{-}^{n}$. We denote the class of agent $i$ 's preferences exhibiting negative income effect by $U_{--}^{i}$, and $U_{--}=U_{--}^{1} \times \cdots \times U_{--}^{n}$.

In Example 1 of nonlinear cost of payments, $U_{L}^{i} \subseteq\left\{u^{i}\left(\cdot, \cdot ; v^{i}, I^{i}\right): I^{i} \in \mathbb{R} \cup\{\infty\}, v^{i} \in\right.$ $\left.\mathbb{R}_{++}\right\} \subseteq U_{+}^{i}$. In Exampl 2 of surplus depending values, given $v^{i}: \mathbb{R} \rightarrow \mathbb{R}$ and $I^{i} \in \mathbb{R}$, compensating transfer $C T^{i}\left(u^{i}, t^{i}\right)$ is the value such that $u^{i}\left(1, t^{i} ; u^{i}, I^{i}\right)=v^{i}\left(I^{i}-t^{i}\right)+$ $I^{i}-t^{i}=u^{i}\left(0, t^{i}-C T^{i}\left(u^{i}, t^{i}\right) ; v^{i}, I^{i}\right)=I^{i}-t^{i}+C T^{i}\left(u^{i}, t^{i}\right)$, i.e., compensating transfer $C T^{i}\left(u^{i}, t^{i}\right)$ coincides with the value $v^{i}\left(I^{i}-t^{i}\right)$. Denote the set of continuous, positive and increasing value functions by $V_{++}^{i}$, the set of continuous, positive and deccreasing value functions by $V_{--}^{i}$, and the set of positive and constant value functions by $V_{0}^{i}$. Then, since $C T^{i}\left(u^{i}, t^{i}\right)=v^{i}\left(I^{i}-t^{i}\right)$ for all $t^{i} \in \mathbb{R},\left\{u^{i}\left(\cdot, \cdot ; v^{i}, I^{i}\right): I^{i} \in \mathbb{R}, v^{i} \in V_{++}^{i}\right\}=U_{++}^{i}$, $\left\{u^{i}\left(\cdot, \cdot ; v^{i}, I^{i}\right): I^{i} \in \mathbb{R}, v^{i} \in V_{++}^{i}\right\}=U_{--}^{i}$, and $\left\{u^{i}\left(\cdot, \cdot ; v^{i}, I^{i}\right): I^{i} \in \mathbb{R}, v^{i} \in V_{0}^{i}\right\}=U_{L}^{i}$.

A preference profile is an $n$-tuple $u=\left(u^{1}, \ldots, u^{n}\right)$ of agents' preferences. A class $U=U^{1} \times \cdots \times U^{n}$ of preference profiles is a set such that $U=U^{1} \times \cdots \times U^{n} \subseteq U_{C}$. The sets $U_{+}, U_{++}, U_{-}, U_{--}$, and $U_{L}$ are the examples of the class of preference profiles.

DeFinition: Given a class $U$ of preference profiles, an (allocation) rule on $U$ is a function $f$ from $U$ to $Z$.

Given $N^{\prime} \subseteq N$, let $U^{N^{\prime}}=\prod_{j \in N^{\prime}} U^{j}$. We denote generic elements of $U, U^{N^{\prime}}$ and $U^{-i}$ by $u, u^{N^{\prime}}$ and $u^{-i}$ respectively. If $u=\left(u^{1}, \ldots, u^{n}\right) \in U, N^{\prime} \subseteq N$, and $i \in N$ are given in advance, $u^{N^{\prime}}$ denotes $\left(u^{j}\right)_{j \in N^{\prime}}$ and $u^{-i}$ denotes $\left(u^{j}\right)_{j \in N \backslash\{i\}}$.

Given a rule $f: U \rightarrow Z$ and $u \in U$, we denote agent $i$ 's outcome consumption of the good by $f_{a}^{i}(u)$, and his outcome payment by $f_{t}^{i}(u)$, and we write:

$$
f(u)=\left(f_{a}^{1}(u), \ldots, f_{a}^{n}(u) ; f_{t}^{1}(u), \ldots, f_{t}^{n}(u)\right), \quad f^{i}(u)=\left(f_{a}^{i}(u), f_{t}^{i}(u)\right), \quad f^{-i}(u)=f(u)_{j \neq i} .
$$

Pareto-efficiency defined below takes the auctioneer's preference into account but assumes that he values the auctioned good at zero.

Definition: An allocation $z \in Z$ is Pareto-efficient for $u \in U$ if there is no allocation $\widehat{z} \in Z$ such that $\sum_{i \in N} \widehat{t^{i}} \geq \sum_{i \in N} t^{i}, u^{i}\left(\widehat{z}^{i}\right) \geq u^{i}\left(z^{i}\right)$ for all $i \in N$, and $u^{i}\left(\widehat{z}^{i}\right)>u^{i}\left(z^{i}\right)$ for some $i \in N$. An allocation rule $f$ is Pareto-efficient if $f(u)$ is efficient for any $u \in U$. 
Remark: An allocation $z$ is Pareto-efficient for $u \in U$ if and only if there is no allocation $\widehat{z} \in Z$ such that $\sum_{i \in N} \widehat{t}^{i}>\sum_{i \in N} t^{i}$, and $u^{i}\left(\widehat{z}^{i}\right) \geq u^{i}\left(z^{i}\right)$ for all $i \in N$.

Definition: A rule $f$ is strategy-proof if for any $u \in u$, any $i \in N$, and any $\widehat{u}^{i} \in U^{i}$,

$$
u^{i}\left(f^{i}(u)\right) \geq u^{i}\left(f^{i}\left(\widehat{u}^{i}, u^{-i}\right)\right) .
$$

Definition: A rule $f$ is individually rational if for any $u \in U$ and any $i \in N$,

$$
u^{i}\left(f^{i}(u)\right) \geq u^{i}(0,0)
$$

Definition: A rule $f$ satisfies nonnegative payment if for any $u \in U$ and any $i \in N$, $f_{t}^{i}(u) \geq 0$.

Given $u \in U$, we rank agents' values, $W P\left(u^{i}, 0\right), i \in N$, of willing to pay, and denote the agent with 1 st highest willing to pay by $i(u, 1)$, the agent with 2 nd highest willing to pay by $i(u, 2)$, and so on. Ties are broken arbitrarily. Under the Vickrey allocation rule defined below, agents with $m$ highest values of willing to pay receive the goods and pay the $(m+1)$-th highest value, $W P\left(u^{i(u, m+1)}, 0\right)$, and other agents pay nothing.

Definition: The Vickrey allocation rule is a rule $g$ such that for any $u \in U$,

$$
\begin{gathered}
g_{a}(u) \in \arg \max \left\{\sum_{i \in N} a^{i} \cdot W P\left(u^{i}, 0\right): a=\left(a^{i}\right)_{i \in N} \in A\right\}, \text { and } \\
g_{t}^{i}(u)= \begin{cases}W P\left(u^{i(u, m+1)}, 0\right) & \text { if } g_{a}^{i}(u)=1, \text { and } \\
0 & \text { otherwise }\end{cases}
\end{gathered}
$$

To be precise, the Vickrey allocation rule is not unique, since the way to break tie is not unique. In other words, there are as many Vickrey allocation rules as the ways to break ties. However, in view of Remark below, we treat a Vickrey allocation rule as if it is unique.

REMARK: Let $g$ and $\hat{g}$ be Vickrey rules. Then

(i) $\sum_{i \in N} g_{t}^{i}(u)=\sum_{i \in N} \hat{g}_{t}^{i}(u)$ for any $u \in U$.

(ii) $u^{i}\left(g^{i}(u)\right)=u^{i}\left(\hat{g}^{i}(u)\right)$ for any $u \in U$ and any $i \in N$.

The following theorems characterize the Vickrey allocation rule as the unique allocation rule satisfying Pareto-efficiency, strategy-proofness, nonnegative payment, and individual rationality on various domains.

THEOREM 1: Let $U$ be a superset of the class $U_{L}$ of quasi-linear preferences and be a subset of the class $U_{C}$ of classical preferences, that is, $U_{L} \subseteq U \subseteq U_{C}$. The Vickrey allocation rule is the unique allocation rule that satisfies Pareto-efficiency, strategy-proofness, nonnegative payment, and individual rationality on $U$.

Theorem 1 implies the characterizations of the Vickrey allocation rule on the class $U_{L}$ of quasi-linear preferences, the class $U_{+}$of preferences exhibiting non-negative income effect, the class $U_{-}$of preferences exhibiting non-positive income effect, etc. Theorem 1 can be also applied to the situation described in Example 1. However, Theorem 1 cannot be applied to the class $U_{++}$of preferences exhibiting positive income effect or the class $U_{--}$of preferences exhibiting negative income effect since these domains do not include $U_{L}$. Theorems 2 and 3 characterize the Vickrey allocation rules on the two domains. 
TheOREM 2: The Vickrey allocation rule is the unique allocation rule that satisfies Pareto-efficiency, strategy-proofness, nonnegative payment, and individual rationality on the class $U_{++}$of preferences exhibiting positive income effect.

THEOREM 3: The Vickrey allocation rule is the unique allocation rule that satisfies Pareto-efficiency, strategy-proofness, nonnegative payment, and individual rationality on the class $U_{--}$of preferences exhibiting negative income effect.

A mechanism is a pair $(S, h)$ of an action space $S$ and an outcome function $h$ such that $S=S^{1} \times \cdots \times S^{n}, S^{i}$ is agent $i$ 's action space, and $h$ is a function from $S$ to $Z$. A mechanism $(S, h)$ implements an allocation rule $f: U \rightarrow Z$ in dominant strategies if there is a dominant strategy $s^{i}: U^{i} \rightarrow S^{i}$ for each agent $i \in N$ and $h(s(u))=f(u)$ for all $u \in U$, where $s(u)=\left(s^{1}\left(u^{1}\right), \ldots, s^{n}\left(u^{n}\right)\right)$. A (closed) auction mechanism is a mechanism $(S, h)$ such that $S^{i}=\mathbb{R}_{+}$for all $i \in N$, and agents who choose $m$ highest values of $s^{i}$ receive the goods. In auction mechanisms, an action $a^{i} \in S^{i}=\mathbb{R}_{+}$is called a "bid".

The Vickrey auction is the auction mechanism such that agents receiving the goods pay the $(m+1)$-th highest bid, and others pay nothing. In the Vickrey auction, it is a dominant strategy for each agent to bid his willing to pay $W P\left(u^{i}, 0\right)$. Thus, the Vickrey auction implements the Vickrey allocation rule in dominant strategies.

\section{Proof of Theorems}

This section is devoted to the proofs of Theorems 1, 2 and 3. First we prove that the Vickrey rule $g$ satisfies efficiency, strategy-proofness, nonnegative payment and individual rationality on the class $U_{C}$ of classical preferences (Proposition 1). This result implies that the Vickrey rule also satisfies the four properties on any subclass $U$ of $U_{C}$. The proofs of our three theorems have the same structure. Thus, we prove only Theorem 1 in detail, and explain how Theorem 1's proof needs to be modified for Theorems 2 and 3.

Proposition 1: The Vickrey rule g satisfies efficiency, strategy-proofness, nonnegative payment and individual rationality on the class $U_{C}$ of classical preferences.

Proof of Proposition 1: Since individual rationality and nonnegative payment are trivial, we show efficiency and strategy-proofness only.

Pareto-Efficiency. Let $u \in U_{C}$. By contradiction, suppose that there is an allocation $z$ that Pareto-dominates $g(u)$. Without loss of generality, assume that $W P\left(u^{1}, 0\right) \geq \cdots \geq$ $W P\left(u^{n}, 0\right)$. Let $N_{0}=\left\{i \in N: g_{a}^{i}(u)=z_{a}^{i}=0\right\}, N_{1}=\left\{i \in N: g_{a}^{i}(u)=1 \& z_{a}^{i}=0\right\}$, $N_{2}=\left\{i \in N: g_{a}^{i}(u)=0 \& z_{a}^{i}=1\right\}$, and $N_{3}=\left\{i \in N: g_{a}^{i}(u)=z_{a}^{i}=1\right\}$. Note that $\sum_{i \in N} g_{t}^{i}(u)=m \cdot W P\left(u^{m+1}, 0\right)$. Also note that for all $i \in N$, if $u^{i}\left(z^{i}\right) \geq u^{i}\left(g^{i}(u)\right)$,

$$
\begin{aligned}
& i \in N_{0} \Rightarrow z_{t}^{i} \leq 0 \\
& i \in N_{1} \Rightarrow z_{t}^{i} \leq 0 \\
& i \in N_{2} \Rightarrow z_{t}^{i} \leq W P\left(u^{i}, 0\right) \\
& i \in N_{3} \Rightarrow z_{t}^{i} \leq g_{t}^{i}(u)=W P\left(u^{m+1}, 0\right)
\end{aligned}
$$

and if $u^{i}\left(z^{i}\right)>u^{i}\left(g^{i}(u)\right)$, the inequalities above are strict. Since $z$ Pareto-dominates $g(u)$, $\sum_{i \in N} g_{t}^{i}(u) \leq \sum_{i \in N} z_{t}^{i}, u^{i}\left(z^{i}\right) \geq u^{i}\left(f^{i}(u)\right)$ for all $i \in N$, and $u^{i}\left(z^{i}\right)>u^{i}\left(f^{i}(u)\right)$ for some 
$i \in N$. Thus, we have

$$
\begin{aligned}
\sum_{i \in N} z_{t}^{i} & <\sum_{i \in N_{2}} W P\left(u^{i}, 0\right)+\left(\# N_{3}\right) \cdot W P\left(u^{m+1}, 0\right) \\
& \leq\left(\# N_{2}+\# N_{3}\right) \cdot W P\left(u^{m+1}, 0\right) \\
& \leq m \cdot W P\left(u^{m+1}, 0\right)=\sum_{i \in N} g_{t}^{i}(u) .
\end{aligned}
$$

This contradicts to $\sum_{i \in N} g_{t}^{i}(u) \leq \sum_{i \in N} z_{t}^{i}$.

Strategy-Proofness. Let $u \in U_{C}, i \in N$, and $\widehat{u}^{i} \in U_{C}^{i}$. We show $u^{i}\left(g^{i}(u)\right) \geq u^{i}\left(g^{i}\left(\widehat{u}^{i}, u^{-i}\right)\right)$. Let $N_{1}=\left\{j \in N: g_{a}^{i}(u)=1\right\}$ and $N_{0}=\left\{j \in N: g_{a}^{i}(u)=0\right\}$.

Case 1: $i \in N_{1}$. Note that $g_{t}^{i}(u)=\max \left\{W P\left(u^{j}, 0\right): j \in N_{0}\right\}$. By individual rationality, $u^{i}\left(g^{i}(u)\right) \geq u^{i}(0,0)$. If $W P\left(\widehat{u}^{i}, 0\right)>g_{t}^{i}(u)$, then $g^{i}\left(\widehat{u}^{i}, u^{-i}\right)=g^{i}(u)$, and so $u^{i}\left(g^{i}\left(\widehat{u}^{i}, u^{-i}\right)\right)=u^{i}\left(g^{i}(u)\right)$. If $W P\left(\widehat{u}^{i}, 0\right)<g_{t}^{i}(u)$, then $g^{i}\left(\widehat{u}^{i}, u^{-i}\right)=0$, and so $u^{i}\left(g^{i}\left(\widehat{u}^{i}, u^{-i}\right)\right)=$ $u^{i}(0,0) \leq u^{i}\left(g^{i}(u)\right)$. If $W P\left(\widehat{u}^{i}, 0\right)=g_{t}^{i}(u)$ and $g_{a}^{i}\left(\widehat{u}^{i}, u^{-i}\right)=1$, then $g^{i}\left(\widehat{u}^{i}, u^{-i}\right)=g^{i}(u)$, and so $u^{i}\left(g^{i}\left(\widehat{u}^{i}, u^{-i}\right)\right)=u^{i}\left(g^{i}(u)\right)$. If $W P\left(\widehat{u}^{i}, 0\right)=g_{t}^{i}(u)$, and $g_{a}^{i}\left(\widehat{u}^{i}, u^{-i}\right)=0$, then $u^{i}\left(g^{i}\left(\widehat{u}^{i}, u^{-i}\right)\right)=u^{i}(0,0) \leq u^{i}\left(g^{i}(u)\right)$.

Case 2: $i \in N_{0}$. Note that $g^{i}(u)=(0,0)$, and $W P\left(u^{i}, 0\right) \leq \min \left\{W P\left(u^{j}, 0\right): j \in N_{1}\right\}$. If $W P\left(\widehat{u}^{i}, 0\right)<\min \left\{W P\left(u^{j}, 0\right): j \in N_{1}\right\}$, then $g^{i}\left(\widehat{u}^{i}, u^{-i}\right)=(0,0)$, and so $u^{i}\left(g^{i}\left(\widehat{u}^{i}, u^{-i}\right)\right)=$ $u^{i}\left(g^{i}(u)\right)$. If $W P\left(\widehat{u}^{i}, 0\right)>\min \left\{W P\left(u^{j}, 0\right): j \in N_{1}\right\}$, then $g^{i}\left(\widehat{u}^{i}, u^{-i}\right)=\left(1, \min \left\{W P\left(u^{j}, 0\right):\right.\right.$ $\left.\left.j \in N_{1}\right\}\right)$, and so by $W P\left(u^{i}, 0\right) \leq \min \left\{W P\left(u^{j}, 0\right): j \in N_{1}\right\}, u^{i}\left(g^{i}\left(\widehat{u}^{i}, u^{-i}\right)\right) \leq u^{i}\left(g^{i}(u)\right)$. If $W P\left(\widehat{u}^{i}, 0\right)=\min \left\{W P\left(u^{j}, 0\right): j \in N_{1}\right\}$, then $u^{i}\left(g^{i}\left(\widehat{u}^{i}, u^{-i}\right)\right)=u^{i}\left(g^{i}(u)\right)$ whether $g_{a}^{i}\left(\widehat{u}^{i}, u^{-i}\right)=0$ or $g_{a}^{i}\left(\widehat{u}^{i}, u^{-i}\right)=1$.

We have established Proposition 1.

\section{Proof OF TheOREM 1:}

Owing to Proposition 1, we only need to show uniqueness. Let $U_{L} \subseteq U=U^{1} \times \cdots \times U^{n} \subseteq U_{C}$. Let $f$ be a rule on $U$ that satisfies Pareto-efficiency, strategy-proofness, individual rationality and nonnegative payment. We prove $f=g$. Fact 1.1 below directly follows from individual rationality and nonnegative payment.

FACT 1.1: For all $i \in N$ and all $u \in U$, if $f_{a}^{i}(u)=0, f_{t}^{i}(u)=0$, and if $f_{a}^{i}(u)=1$, $f_{t}^{i}(u) \leq W P\left(u^{i}, 0\right)$.

FACT 1.2: For all $i, j \in N$ and all $u \in U$,

$$
\left[W P\left(u^{i}, 0\right)<W P\left(u^{j}, 0\right), u^{i} \in U_{L}, \text { and } f_{a}^{i}(u)=1\right] \Rightarrow f_{a}^{j}(u)=1 \text {. }
$$

Proof of FACT 1.2: By contradiction, suppose $f_{a}^{j}(u)=0$. By Fact $1.1, f_{t}^{j}(u)=0$. Fact 1.1 also implies $f_{t}^{i}(u) \leq W P\left(u^{i}, 0\right)$. Then the allocation $\widehat{z}$ such that $\widehat{z}^{i}=\left(0, f_{t}^{i}(u)-\right.$ $\left.W P\left(u^{i}, 0\right)\right), \widehat{z}^{j}=\left(1, W P\left(u^{j}, 0\right)\right)$, and $\widehat{z}^{k}=f^{k}(u)$ for all $k \neq i, j$ Pareto-dominates $f(u)$ since $\sum_{k \in N} \widehat{z}^{k}=\sum_{k \in N} f^{k}(u)+W P\left(u^{j}, 0\right)-W P\left(u^{i}, 0\right) .{ }^{10}$ This is a contradiction to Pareto-efficiency.

FACT 1.3: For all $i \in N$ and all $u \in U$, if $f_{a}^{i}(u)=1, f_{t}^{i}(u) \geq W P\left(u^{i(u, m+1)}, 0\right)$.

Proof OF FACT 1.3: Suppose the contrary so that $f_{a}^{i}(u)=1$ and $f_{t}^{i}(u)<W P\left(u^{i(u, m+1)}, 0\right)$ for some $i \in N$ and $u \in U$. Let $\widehat{u}^{i} \in U_{L}$ be such that $f_{t}^{i}(u)<W P\left(\widehat{u}^{i}, 0\right)<W P\left(u^{i(u, m+1)}, 0\right)$.

\footnotetext{
${ }^{10}$ It may be the case that agent $i$ 's payment $f_{t}^{i}(u)-W P\left(u^{i}, 0\right)$ of the allocation $\widehat{z}$ is negative. Such an allocation is admissible since we only assume the nonnegative payment of the allocation rule $f$.
} 
If $f_{a}^{i}\left(\widehat{u}^{i}, u^{-i}\right)=1$, then by Fact 1.2 , for all $j \in N$ with $W P\left(u^{j}, 0\right) \geq W P\left(u^{i(u, m+1)}, 0\right)$, $f_{a}^{j}\left(\widehat{u}^{i}, u^{-i}\right)=1$. This implies $\sum_{j \in N} f_{a}^{j}\left(\widehat{u}^{i}, u^{-i}\right)>m$, contradicting feasibility. Thus, we have $f_{a}^{i}\left(\widehat{u}^{i}, u^{-i}\right)=0$, and so by Fact $1.1, f_{t}^{i}\left(\widehat{u}^{i}, u^{-i}\right)=0$. Strategy-proofness then implies $0=\widehat{u}^{i}\left(f^{i}\left(\widehat{u}^{i}, u^{-i}\right)\right) \geq \widehat{u}^{i}\left(f^{i}(u)\right)=W P\left(\widehat{u}^{i}, 0\right)-f_{t}^{i}(u)$, i.e., $f_{t}^{i}(u) \geq W P\left(\widehat{u}^{i}, 0\right)$. This is a contradiction to $f_{t}^{i}(u)<W P\left(\widehat{u}^{i}, 0\right)$.

Q.E.D.

FACT 1.4: For all $i \in N$ and all $u \in U$,

$$
W P\left(u^{i}, 0\right)=W P\left(u^{i(u, m+1)}, 0\right) \Rightarrow u^{i}\left(f^{i}(u)\right)=u^{i}(0,0) .
$$

Proof of FACT 1.4: If $f_{a}^{i}(u)=0$, then the result follows from Fact 1.1. Let $f_{a}^{i}(u)=1$. Since $W P\left(u^{i}, 0\right)=W P\left(u^{i(u, m+1)}, 0\right)$, Fact 1.1 and Fact 1.3 imply $W P\left(u^{i(u, m+1)}, 0\right) \geq$ $f_{t}^{i}(u) \geq W P\left(u^{i(u, m+1)}, 0\right)$. Thus we have $f_{t}^{i}(u)=W P\left(u^{i(u, m+1)}, 0\right)$. Therefore, $u^{i}\left(f^{i}(u)\right)=$ $u^{i}(0,0)$.

Q.E.D.

Let $u \in U$ be given. Without loss of generality, we assume that $W P\left(u^{1}, 0\right) \geq \cdots \geq$ $W P\left(u^{n}, 0\right)$, and we define the sets $N_{+}, N_{0}$, and $N_{-}$by

$$
\begin{aligned}
& N_{+}=\left\{i \in N: W P\left(u^{i}, 0\right)>W P\left(u^{m+1}, 0\right)\right\}, \\
& N_{0}=\left\{i \in N: W P\left(u^{i}, 0\right)=W P\left(u^{m+1}, 0\right)\right\}, \text { and } \\
& N_{-}=\left\{i \in N: W P\left(u^{i}, 0\right)<W P\left(u^{m+1}, 0\right)\right\} .
\end{aligned}
$$

In order to show that $f$ is a Vickrey rule, we have only to show that

$$
\begin{aligned}
& f^{i}(u)= \begin{cases}\left(1, W P\left(u^{m+1}, 0\right)\right) & \text { if } i \in N_{+}, \\
(0,0) & \text { if } i \in N_{-}, \text {and }\end{cases} \\
& u^{i}\left(f^{i}(u)\right)=u^{i}(0,0) \\
& \text { if } i \in N_{0} .
\end{aligned}
$$

FACT 1.5: For all $i \in N_{-}, f^{i}(u)=(0,0)$.

Proof of FACT 1.5: Let $i \in N_{-}$. Suppose on the contrary $f_{a}^{i}(u)=1$. By Facts 1.1 and 1.3, $W P\left(u^{m+1}, 0\right) \leq f_{t}^{i}(u) \leq W P\left(u^{i}, 0\right)$. This is a contradiction to $i \in N_{-}$. Thus $f_{a}^{i}(u)=0$. Then, Facts 1.1 implies $f^{i}(u)=(0,0)$.

Q.E.D.

FACT 1.6: For all $i \in N_{+}, f^{i}(u)=\left(1, W P\left(u^{m+1}, 0\right)\right)$.

PROOF OF FACT 1.6: We proceed this proof by two steps.

Step 1: $f_{a}^{i}(u)=1$ for all $i \in N_{+}$. Suppose on the contrary that there is $i \in N_{+}$ such that $f_{a}^{i}(u)=0$. By Fact 1.1, $f_{t}^{i}(u)=0$. Note that there is $j \in N_{0} \cup N_{-}$such that $f_{a}^{j}(u)=1$. Fact 1.5 implies $j \in N_{0}$. Moreover, Fact 1.4 implies $f_{t}^{j}(u)=W P\left(u^{m+1}, 0\right)$. Then the allocation $\widehat{z}$ such that $\widehat{z}^{i}=\left(1, W P\left(u^{i}, 0\right)\right), \widehat{z}^{j}=(0,0)$, and $\widehat{z}^{k}=f^{k}(u)$ for all $k \neq$ $i, j$ Pareto-dominates $f(u)$ since $\sum_{k \in N} \widehat{z}^{k}=\sum_{k \in N} f^{k}(u)+W P\left(u^{i}, 0\right)-W P\left(u^{m+1}, 0\right)>$ $\sum_{k \in N} f^{k}(u)$. This is a contradiction.

Step 2: $f_{t}^{i}(u)=W P\left(u^{m+1}, 0\right)$ for all $i \in N_{+}$. Let $i \in N_{+}$. By Step 1, $f_{a}^{i}(u)=1$. Fact 1.3 implies $f_{t}^{i}(u) \geq W P\left(u^{m+1}, 0\right)$. By contradiction, suppose that $f_{t}^{i}(u)>W P\left(u^{m+1}, 0\right)$. Let $\widehat{u}^{i} \in U_{L}$ be such that $f_{t}^{i}(u)>W P\left(\widehat{u}^{i}, 0\right)>W P\left(u^{m+1}, 0\right)$. Then, Step 1 implies $f_{a}^{i}\left(\widehat{u}^{i}, u^{-i}\right)=1$. Thus by Fact $1.1, f_{t}^{i}\left(\widehat{u}^{i}, u^{-i}\right) \leq W P\left(\widehat{u}^{i}, 0\right)<f_{t}^{i}(u)$. This is a contradiction to strategy-proofness.

Q.E.D. 
Fact 1.4, Fact 1.5, and Fact 1.6 imply that $f$ is a Vickrey rule.

We have established Theorem 1.

\section{ProOFs OF THEOREMS 2 AND 3:}

Let $f$ be a rule on $U_{++}$that satisfies Pareto-efficiency, strategy-proofness, individual rationality and nonnegative payment to prove Theorem 2. (Let $f$ be a rule on $U_{--}$that satisfies Pareto-efficiency, strategy-proofness, individual rationality and nonnegative payment to prove Theorem 3.) We prove $f=g$. Facts 2.1 and 3.1 below are the counterparts of Fact 1 on $U_{++}$and $U_{--}$respectively. They directly follow from individual rationality and nonnegative payment.

FACT 2.1: For all $i \in N$ and all $u \in U_{++}$, if $f_{a}^{i}(u)=0, f_{t}^{i}(u)=0$, and if $f_{a}^{i}(u)=1$, $f_{t}^{i}(u) \leq W P\left(u^{i}, 0\right)$.

FACT 3.1: For all $i \in N$ and all $u \in U_{--}$, if $f_{a}^{i}(u)=0, f_{t}^{i}(u)=0$, and if $f_{a}^{i}(u)=1$, $f_{t}^{i}(u) \leq W P\left(u^{i}, 0\right)$.

Facts 2.2 and 3.2 below are the counterparts of Fact 1.2 on $U_{++}$and $U_{--}$respectively.

FACT 2.2: For all $i, j \in N$ and all $u \in U_{++}$,

$$
\left[f_{a}^{i}(u)=1, \text { and } \forall t^{i} \in\left[0, W P\left(u^{i}, 0\right)\right], C T\left(u^{i}, t^{i}\right)<W P\left(u^{j}, 0\right)\right] \Rightarrow f_{a}^{j}(u)=1 .
$$

FACT 3.2: For all $i, j \in N$ and all $u \in U_{--}$,

$$
\left[f_{a}^{i}(u)=1, \text { and } \forall t^{i} \in\left[0, W P\left(u^{i}, 0\right)\right], C T\left(u^{i}, t^{i}\right)<W P\left(u^{j}, 0\right)\right] \Rightarrow f_{a}^{j}(u)=1 .
$$

Proof of FACT 2.2: By contradiction, suppose that $f_{a}^{j}(u)=0$. By Fact $2.1, f_{t}^{j}(u)=0$. Moreover, Fact 2.1 together with nonnegative payment implies $0 \leq f_{t}^{i}(u) \leq W P\left(u^{i}, 0\right)$, so that $C T\left(u^{i}, f_{t}^{i}(u)\right)<W P\left(u^{j}, 0\right)$. Then the allocation $\widehat{z}$ such that $\widehat{z}^{i}=\left(0, f_{t}^{i}(u)-\right.$ $\left.C T\left(u^{i}, f_{t}^{i}(u)\right)\right), \widehat{z}^{j}=\left(1, W P\left(u^{j}, 0\right)\right)$, and $\widehat{z}^{k}=f^{k}(u)$ for all $k \neq i, j$ Pareto-dominates $f(u)$ since $\left.\sum_{k \in N} \widehat{z}^{k}=\sum_{k \in N} f^{k}(u)+W P\left(u^{j}, 0\right)-C T\left(u^{i}, f_{t}^{i}(u)\right)\right)>\sum_{k \in N} f^{k}(u)$. This is a contradiction to Pareto-efficiency.

Q.E.D.

The proof of Fact 3.2 is the same as Fact 2.2 except that Fact 2.1 is replaced by Fact 3.1 .

Facts 2.3 and 3.3 below are the counterparts of Fact 1.3 on $U_{++}$and $U_{--}$respectively.

FACT 2.3: For all $i \in N$ and all $u \in U_{++}$, if $f_{a}^{i}(u)=1, f_{t}^{i}(u) \geq W P\left(u^{i(u, m+1)}, 0\right)$.

FACT 3.3: For all $i \in N$ and all $u \in U_{--}$, if $f_{a}^{i}(u)=1, f_{t}^{i}(u) \geq W P\left(u^{i(u, m+1)}, 0\right)$.

Proof OF FACT 2.3: Suppose the contrary so that $f_{a}^{i}(u)=1$ and $f_{t}^{i}(u)<W P\left(u^{i(u, m+1)}, 0\right)$ for some $i \in N$ and $u \in U_{++}$. Let $\widehat{u}^{i} \in U_{++}^{i}$ be such that $f_{t}^{i}(u)<W P\left(\widehat{u}^{i}, 0\right)<$ $W P\left(u^{i(u, m+1)}, 0\right)$, and for all $t^{i} \in\left[0, W P\left(u^{i}, 0\right)\right]$ and for all $j \neq i$ with $W P\left(u^{j}, 0\right) \geq$ $W P\left(u^{i(u, m+1)}, 0\right), C T\left(\widehat{u}^{i}, t^{i}\right)<W P\left(u^{j}, 0\right)$. If $f_{a}^{i}\left(\widehat{u}^{i}, u^{-i}\right)=1$, then by Fact 2.2 , for all $j \in$ $N$ with $W P\left(u^{j}, 0\right) \geq W P\left(u^{i(u, m+1)}, 0\right), f_{a}^{j}\left(\widehat{u}^{i}, u^{-i}\right)=1$. This implies $\sum_{j \in N} f_{a}^{j}\left(\widehat{u}^{i}, u^{-i}\right)>$ $m$, contradicting feasibility. Thus, we have $f_{a}^{i}\left(\widehat{u}^{i}, u^{-i}\right)=0$, and so by Fact $2.1, f_{t}^{i}\left(\widehat{u}^{i}, u^{-i}\right)=$ 0 . The definition of $W P$ and strategty-proofness then imply $\widehat{u}^{i}\left(1, W P\left(\widehat{u}^{i}, 0\right)\right)=\widehat{u}^{i}(0,0)=$ $\widehat{u}^{i}\left(f^{i}\left(\widehat{u}^{i}, u^{-i}\right)\right) \geq \widehat{u}^{i}\left(f^{i}(u)\right)=\widehat{u}^{i}\left(1, f_{t}^{i}(u)\right)$. Thus, we have $W P\left(\widehat{u}^{i}, 0\right) \leq f_{t}^{i}(u)$. This is a contradiction to $f_{t}^{i}(u)<W P\left(\widehat{u}^{i}, 0\right)$.

Q.E.D.

The proof of Fact 3.3 is the same as Fact 2.3 except that Fact 2.1, Fact 2.2, $U_{++}$and $U_{++}^{i}$ are replaced by Fact Fact 3.1, Fact 3.2, $U_{--}$and $U_{--}^{i}$ respectively. 
Facts 2.4 and 3.4 below are the counterparts of Fact 1.4 on $U_{++}$and $U_{--}$respectively. Similarly to Fact 1.4, Fact 2.4 follows from Facts 2.1 and 2.3, and Fact 3.4 follows from Facts 3.1 and 3.3.

FACT 2.4: For all $i \in N$ and all $u \in U_{++}$, if $W P\left(u^{i}, 0\right)=W P\left(u^{i(u, m+1)}, 0\right), u^{i}\left(f^{i}(u)\right)=$ $u^{i}(0,0)$.

FACT 3.4: For all $i \in N$ and all $u \in U_{--}$, if $W P\left(u^{i}, 0\right)=W P\left(u^{i(u, m+1)}, 0\right), u^{i}\left(f^{i}(u)\right)=$ $u^{i}(0,0)$.

Let $u \in U_{++}$be given to prove Theorem 2. (Let $u \in U_{--}$be given to prove Theorem 3.) Without loss of generality, we assume that $W P\left(u^{1}, 0\right) \geq \cdots \geq W P\left(u^{n}, 0\right)$, and we define the sets $N_{+}, N_{0}$, and $N_{-}$as in the proof of Theorem 1 .

Similarly to Fact 1.5, Fact 5 below directly follows from Facts 2.1 and 2.3 in the case of $u \in U_{++}$. (Fact 5 follows from Facts 3.1 and 3.3 in the case of $u \in U_{--}$.)

FACT 5: For all $i \in N_{-}, f^{i}(u)=(0,0)$.

Similarly to Fact 1.6, Fact 6 below follows from Facts 2.1, 2.3, 2.4 and 2.6 in the case of $u \in U_{++}$. (Fact 6 follows from Facts 2.1, 2.3, 2.4 and 2.6 in the case of $u \in U_{--}$.)

FACT 6: For all $i \in N_{+}, f^{i}(u)=\left(1, W P\left(u^{m+1}, 0\right)\right)$.

Facts $2.4,5$ and 6 imply $f=g$ on $U_{++}$. Facts $3.4,5$ and 6 imply $f=g$ on $U_{--}$.

\section{References}

[1] Che, Y.K., and Gale., "Standard Auction with Finacially Constrained Bidders," Review od Economic Studies, (1998): 65, 1-21.

[2] Chew Soo Hong, and Serizawa, S., "Characterizing Vickrey Auction by Induction Logic," 2004, mimeo

[3] Clarke, Edward H., "Multipart Pricing of Public Goods," Public Choice (1971): 1733

[4] Demange, G., Gale, D., and Sotomayor, M., "Multi-Item Auction," Journal of Political Economy, (1986): 863-872

[5] Green, J., and Laffont, J-J., "Characterization of Satisfactory Mechanisms For the Revelation of Preferences for Public Goods," Econometrica (1977): 427-438

[6] Groves, Theodore, "Incentives in Teams," Econometrica (1973): 617-632

[7] Hatfield, J.W., and Milgrom, P., "Auctions, Matching, and the Law of Aggregate Demand," (2005), American Economic Review, Forthcoming.

[8] Holmstrom, B., "Groves' Scheme on Restricted Domain," Econometrica (1979), 11371144

[9] Klemperer, P., "What really Matters in Auction Design," Journal of Economic Perspectives (2002): 169-189

[10] Krishna, V., "Auction Theory," (2002), Academic Press. 
[11] MacMillan, John, "Selling Spectrum Rights," Journal of Economic Perspectives (1994), 145-162

[12] Makowski, Louise and Ostroy, Joseph, "Vickrey-Clarke-Groves Mechanisms and Perfect Competition," Journal of Economic Theory (1987): 244-261

[13] Miyake, M., "On the incentive properties of multi-item auctions," International Journal of Game Theory (1998), 27 1-19

[14] Maskin, E., and Riley, J., "Optimal Auctions with Risk-Averse Bidders," Econometrica (1984) 52 (6), 1473-1518

[15] Milgrom, Paul, "Putting Auction Theory to Work," (2004) Cambridge University Press.

[16] Roth, A., and Sotomayor, M., "Two-sided matching" (1992) In: Aumann RJ, Hart S (eds) Handbook of Game Theory with Economic Applications, Volume I, NorthHolland, Amsterdam.

[17] Roth, A., and Sotomayor, M., "Two-sided matching: A Study in Game-Theoretic Modeling and Analysis" (1990) Econometric Society Monographs, Cambridge University Press.

[18] Sakai, T., "Second price auctions when preferences are not quasi-linear," (2005) mimeo.

[19] Vickrey, William, Counterspeculation, auctions, and competitive sealed tenders, Journal of Finance (1961), 8-37

[20] Walker, Mark, "A Note on the Characterization of Mechanisms for the Revelation of Preferences," Econometrica (1978): 147-152 